\title{
Review \\ Effect of Resin Infiltration on Enamel: A Systematic Review and Meta-Analysis
}

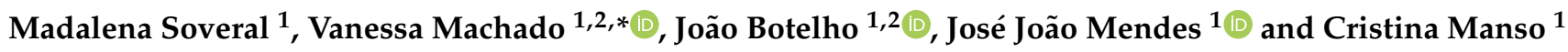 \\ 1 Clinical Research Unit (CRU), Centro de Investigação Interdisciplinar Egas Moniz (CiiEM), \\ Egas Moniz-Cooperativa de Ensino Superior, CRL, 2829-511 Almada, Portugal; \\ madalenasvsoveral@gmail.com (M.S.); jbotelho@egasmoniz.edu.pt (J.B.); jmendes@egasmoniz.edu.pt (J.J.M.); \\ mansocristina@gmail.com (C.M.) \\ 2 Evidence-Based Hub, Clinical Research Unit, Centro de Investigação Interdisciplinar Egas Moniz (CiiEM), \\ Egas Moniz Cooperativa de Ensino Superior, CRL, 2829-511 Almada, Portugal \\ * Correspondence: vmachado@egasmoniz.edu.pt
}

Citation: Soveral, M.; Machado, V.; Botelho, J.; Mendes, J.J.; Manso, C. Effect of Resin Infiltration on Enamel: A Systematic Review and Meta-Analysis. J. Funct. Biomater. 2021, 12, 48. https://doi.org/ $10.3390 /$ jfb12030048

Academic Editors: Neamat Hassan Abubakr and Karl Kingsley

Received: 8 July 2021

Accepted: 12 August 2021

Published: 16 August 2021

Publisher's Note: MDPI stays neutral with regard to jurisdictional claims in published maps and institutional affiliations.

Copyright: (c) 2021 by the authors. Licensee MDPI, Basel, Switzerland. This article is an open access article distributed under the terms and conditions of the Creative Commons Attribution (CC BY) license (https:// creativecommons.org/licenses/by/ $4.0 /)$.

\begin{abstract}
Subsurface enamel demineralization beneath an intact surface layer or white spots lesions (WSL) can and should be treated with non-invasive procedures to impede the development of a cavitated lesion. We aim to analyze if infiltrative resin improves enamel roughness, microhardness, shear bond strength, and penetration depth. MEDLINE [via Pubmed], Cochrane Central Register of Controlled Trials, Embase, Web of Science, Scholar, and LILACS were searched until May 2021. Methodological quality was assessed using the Joanna Briggs Institute Clinical Appraisal Checklist for Experimental Studies. Pairwise ratio of means (ROM) meta-analyses were carried out to compare the enamel properties after treatment with infiltrative resin on sound enamel and WSLs. From a total of 1604 articles, 48 studies were included. Enamel surface roughness decreased 35\% in sound enamel (95\%CI: 0.49-0.85, $\mathrm{I}^{2}=98.2 \%$ ) and 54\% in WSLs (95\%CI: 0.29-0.74, $\mathrm{I}^{2}=98.5 \%$ ). Microhardness reduced $24 \%$ in sound enamel (95\%CI: $0.73-0.80, \mathrm{I}^{2}=99.1 \%$ ) and increased by $68 \%$ in WSLs (95\%CI: $1.51 ; 1.86$, $\left.\mathrm{I}^{2}=99.8 \%\right)$. Shear bond strength reduced of $25 \%$ in sound enamel (95\%CI: $\left.0.60 ; 0.95, \mathrm{I}^{2}=96.9 \%\right)$ and increased by $89 \%$ in WSLs (95\%CI: $1.28-2.79, \mathrm{I}^{2}=99.8 \%$ ). Penetration depth was $65.39 \%$ of the WSLs (95\%CI: 56.11-74.66, $\mathrm{I}^{2}=100 \%$ ). Infiltrative resins effectively promote evident changes in enamel properties in sound and WSLs. Future studies with long-term follow-ups are necessary to corroborate these results from experimental studies.
\end{abstract}

Keywords: resin infiltration; demineralization; white spot lesions; surface roughness; microhardness; shear bond strength; penetration depth

\section{Introduction}

Dental caries is an oral condition estimated to affect 2.4 billion people worldwide in 2010 [1,2], while the frequency of white spot lesions (WSLs) varies between $2 \%$ and 97\% [3-6]. WSLs were firstly described in 1908 by Black [7]. In the last decades, the prevalence of WSLs has increased as a side effect to fixed orthodontic appliances [8-11]. Consequently, multiple approaches have been proposed to prevent, manage and treat dental caries [12-14], while non-invasive therapies have emerged to treat early signs of WSLs [15-17].

Caries infiltration is a minimally invasive technique for the management of smooth surface and proximal non-cavitated caries lesions. Several remineralization products have been presented to this end, such as fluoride, casein phosphopeptide, amorphous calcium phosphate, and microabrasion $[7,8]$. Low-viscosity light-cured resins are another popular approach [9]. The infiltration of resins creates a diffusion barrier inside the enamel lesion body [18], retarding enamel dissolution [10,11], and the retention loss is unlikely to occur [19]. 
Clinical evidence points to the partial or total ability of infiltrative resins to mask enamel whitish discoloration [12], despite its clinical efficacy still warrants long-term confirmation [7,13-17]. A previous systematic review of in vitro studies has shown an increase of surface microhardness of WSLs after resin infiltration, and an opposite result in sound enamel [20]. Notwithstanding, there is still uncertainty regarding its efficacy on other characteristics (namely, surface roughness, shear bond strength, and penetration depth). Hence, appraising the available evidence on these characteristics in a systematic manner becomes clinically relevant to understand the potential of this minimally invasive procedure.

Considering the recent increased number of studies, here we present a systematic review assessing the effect of infiltrative resins on surface roughness, microhardness, shear bond strength and penetration depth in permanent teeth with and without enamel lesions.

\section{Materials and Methods}

\subsection{Protocol and Registration}

We registered and approved this systematic review protocol a priori at the National Institute for Health Research PROSPERO database, International Prospective Register of Systematic Review (Available online: www.crd.york.ac.uk, ID number: CRD42019140860) and we reported the review information according to the PRISMA guidelines [18] (Table S1).

\subsection{Focused Question and Eligibility Criteria}

To answer our main question, we developed a protocol with two PICO questions:

1. "Do infiltrative resin in sound enamel and WSLs improve the surface roughness, microhardness and shear bond strength?" and

2. "What is the penetration depth capacity of the infiltrative resin in WSLs?"

Each question had the following statements:

1. Teeth with sound enamel, and teeth with WSLs or teeth classified with ICDAS 1 or 2 (Population, P); Resin infiltration (Intervention, I); Initial condition or no treatment (Comparison, C); Disappearance or improvement of the surface roughness, microhardness, and shear bond strength (Outcome, O).

2. Teeth with WSLs or teeth classified with ICDAS 1 or 2 (Population, P); Resin infiltration (Intervention, I); Not applicable (Comparison, C); Penetration depth (Outcome, O).

In vitro studies that assess the enamel surface roughness, microhardness, shear bond strength and penetration depth before and after resin infiltration were eligible. In vivo studies were excluded because the methods used for clinical evaluation of those four characteristics mentioned in patients were substantially different from those used on in vitro studies, and there were innumerable variables that we are unable to control, such as the quality of patients' saliva, their cooperation, and the variation of the techniques and analyses performed. Although the teeth are considered healthy, we assume that they have changed their crystalline structure once they have been subjected to home care products, such as fluoridated toothpaste and oral rinsing solutions or mouthwash being subjected to remineralization with the incorporation of fluoride ions.

Regarding color, no assessment was made because Borges et al. in 2017 [21] systematically evaluated this characteristic in patients. Furthermore, editorial, letters, reviews, thesis, case reports, and case series were excluded. Most studies used profilometers (in Ra) to quantify the surface roughness, and therefore studies that quantified enamel surface roughness using other appliances were not included because it does not allow comparison.

\subsection{Search Strategy}

Seven electronic databases (MEDLINE [via Pubmed], Cochrane Central Register of Controlled Trials, Embase, Web of Science, Scholar, and LILACS) were searched systematically until May 2021. The following search strategies were adjusted to each database: ("infiltrative resin" OR "resin infiltration") AND ("white spot lesions" OR "white spots" OR “WSL" OR “Enamel demineralization"). In addition, we search manually in Journal 
of Dentistry, The Journal of Prosthetic Dentistry, Clinical Implant Dentistry and Related Research, Operative Dentistry, Community Dentistry, and Oral Epidemiology, Journal of Conservative Dentistry, and International Journal of Dentistry. The Grey literature was searched using the latter strategy in OpenGray. Any limitation of the publication period and language was applied. Authors were contacted, when necessary, for additional data clarification.

\subsection{Study Process}

Two independent researchers (M.S. and V.M.) screened the title and/or abstract of retrieved studies. Any disagreements were resolved by discussion with a third author (C.M.). The final selection of studies was independently performed by two authors (M.S. and V.M.) who reviewed the selected papers' full text based on the inclusion criteria mentioned above. For measurement reproducibility purposes, inter-examiner reliability following full-text assessment was calculated via kappa statistics.

A predefined table was used to extract necessary data from each eligible study, including the citation, publication status and year of publication, study design, inclusion/exclusion criteria, number of specimens per group, demineralization process, resin infiltration protocol, surface roughness, microhardness, shear bond strength and penetration depth measurement method. Concerning additional data clarifications, we attempted to contact the corresponding authors twice, with an interval time of 1 week.

\subsection{Methodological Quality Assessment}

Two researchers (M.S. and V.M.) independently assessed the methodological quality of the included studies, following the Joanna Briggs Institute Clinical Appraisal Checklist for Experimental Studies. This assessment tool was adapted from previously published systematic reviews [22-24]. The items on the checklist were as follows: (1) clearly mention aim, justification of sample size; (2) sample randomization; (3) blind treatment allocation; (4) possibility of comparison between control and treatment groups; (5) baseline equivalence of control and treatment groups; (6) clearly describe the preparation protocol; (7) clearly report the experimental protocol; (8) measurement method, and adequate statistical analysis. Each item was scored using a 2-point scale: 0-not reported or reported inadequately; and 1-reported and adequate. Any disagreements between the examiners were resolved through discussion with a third author (C.M.).

\subsection{Statistical Analysis}

For continuous data, mean values and standard deviations (SD) were collected to predefined tables prepared to determine the quantity of data. If median and interquartile range were reported in the selected studies, mean and SD were calculated following Hozo's formula [25]. The random-effect meta-analysis and forest plots were calculated in $\mathrm{R}$ version 3.4.1 (R Studio Team 2018) using 'meta' package [26], through DerSimonian-Laird random-effects meta-analysis. Firstly, we started by conducting an a priori sensitivity analysis comparing Standardized Mean Difference (SMD) versus Ratio of Means (RoM) meta-analyses. If there are similar results in terms of heterogeneity and significance, RoM was applied as it would allow easier and direct interpretation of the results (reported as percentage) [26]. To investigate sources of heterogeneity, meta-regression analysis was conducted for method, $\mathrm{pH}$, and demineralization time. $\mathrm{I}^{2}$ index and Cochrane's $\mathrm{Q}$ statistic were used to assess statistical heterogeneity $(p<0.1)$ and $\chi 2$ test calculated overall homogeneity [26]. Substantial heterogeneity was considered when $\mathrm{I}^{2}$ statistics exceeded $50 \%$ [27]. All tests were two-tailed with alpha set at 0.05 except for the homogeneity test whose significance level cutoff was 0.10 due to the low power of the $\chi 2$ test with a limited amount of studies. Overall estimates were reported with $95 \%$ confidence interval (CI). For meta-analysis including 10 or more studies, we analyzed publication bias [28]. 


\section{Results}

\subsection{Study Selection}

The initial database search strategy retrieved 1604 possibly relevant articles. After exclusion of all duplicates, 175 articles were assessed for full paper review eligibility. Among these, 127 articles were excluded with the respective reasons for exclusion detailed in Table S2. A total of 48 articles fulfilled the inclusion criteria and were selected for further quantitative and qualitative analyses (Figure 1). Good inter-examiner agreement was obtained during full-text screening and article final selection (Cohen's Kappa: 0.92; 95\% CI: 0.89; 0.94).

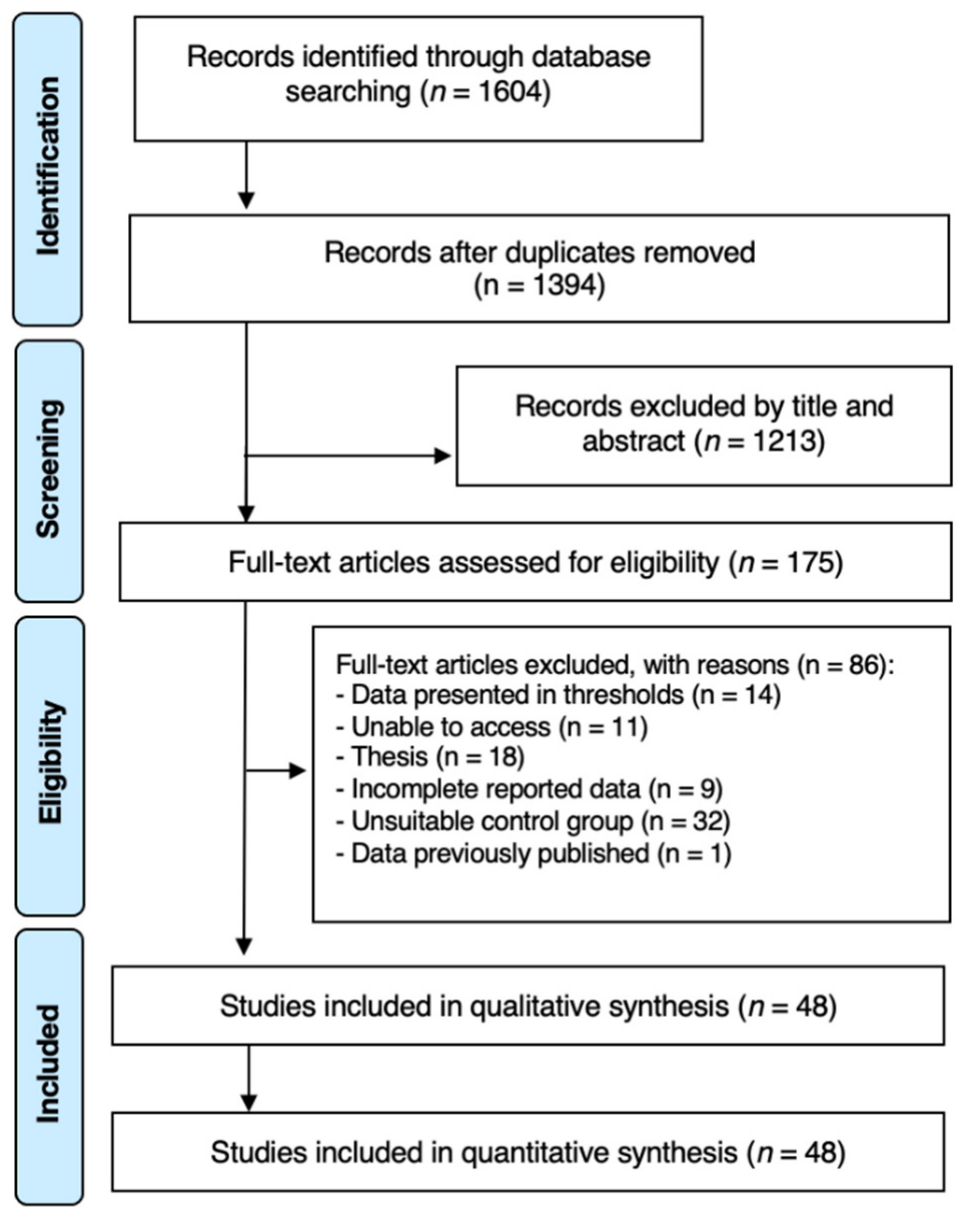

Figure 1. PRISMA flow-chart representing the results of the workflow to identify eligible studies.

\subsection{Characteristics of the Studies}

In this systematic review, twenty-three articles reported information about microhardness [27-49], ten evaluated surface roughness, [27,31,37,47,49-54], sixteen assessed the penetration depth $[40,46,50,55-67]$ and eight explored shear bond strength after resin infiltration [32,52,68-73].

Overall, twelve studies included bovine teeth ( $n$ per group $=803$ ) while twenty-five studies included human teeth $(n$ per group $=865$ ). Nine studies reported data without specimen demineralization previously to resin infiltration $[47,54,55,57,59,61-64,74]$ and thirty-nine articles included the demineralization process before the infiltrative resin $[21,27-44,48-53,56,58,60,65-67,69-73]$. The demineralization process consists of emerging the teeth in a demineralization solution, with $\mathrm{pH}$ between 4 and 5 for a period of time that ranges from $1 \mathrm{~min}$ to $1200 \mathrm{~h}$, to simulate the formation of WSLs (Table 1). 
Nowadays there is only one commercial kit available, ICON ${ }^{\circledR}$ (DMG, Hamburg, Germany), that aims to infiltrate proximal and vestibular lesions [75]. Taking this into account, all studies used the ICON ${ }^{\circledR}$ protocol to do the infiltration of resin.

Table 1. Overview of the Included Studies.

\begin{tabular}{|c|c|c|c|c|c|c|}
\hline Study & Funding & $n$ & Specimen Origin & $\begin{array}{l}\text { Exclusion } \\
\text { CRITERIA }\end{array}$ & $\begin{array}{l}\text { WSLs Preparation } \\
\text { (pH for hours) }\end{array}$ & Outcome Reported \\
\hline $\begin{array}{l}\text { Pancu et al. } 2011 \\
\text { (Romania) [42] }\end{array}$ & NR & 10 & $\begin{array}{l}\text { Human (bicusps or } \\
\text { molars) }\end{array}$ & NR & $\mathrm{pH}: 4.4$ for $120 \mathrm{~h}$ & $\begin{array}{c}\text { Microhardness } \\
\text { (Vicker hardness- special } \\
\text { device for microhardness } \\
\text { testing with a squared } \\
\text { diamond head) }\end{array}$ \\
\hline $\begin{array}{l}\text { Meyer-Lueckel et al. } \\
2011 \text { (Germany) [59] }\end{array}$ & $\begin{array}{c}\text { DFG: PA 1508/1-1. HML } \\
\text { and SP and royalties from } \\
\text { DMG, Hamburg }\end{array}$ & 20 & $\begin{array}{l}\text { Human } \\
\text { (molars and } \\
\text { premolars) }\end{array}$ & $\begin{array}{c}\text { Active } \\
\text { non-cavitated } \\
\text { proximal WSL } \\
\text { (ICDAS code 2) }\end{array}$ & $\begin{array}{c}\text { Without } \\
\text { demineralization }\end{array}$ & $\begin{array}{l}\text { Penetration Depth } \\
\text { (Confocal laser scanning } \\
\text { microscopy (CLSM)) }\end{array}$ \\
\hline $\begin{array}{l}\text { Paris et al. } 2011 \text { B } \\
\text { (Germany) [62] }\end{array}$ & $\begin{array}{l}\text { Institute for Immunology, } \\
\text { UK-SH, } \\
\text { Christian-Albrechts } \\
\text { Universitat zu Kiel for } \\
\text { providing the CLSM. The } \\
\text { Charité-Universitatmedizin } \\
\text { Berlin holds US and } \\
\text { European patents }\end{array}$ & 19 & $\begin{array}{l}\text { Human } \\
\text { (molars and } \\
\text { premolars) }\end{array}$ & $\begin{array}{c}\text { Active } \\
\text { non-cavitated } \\
\text { proximal lesions } \\
\text { scored as ICDAS } 2\end{array}$ & $\begin{array}{c}\text { Without } \\
\text { demineralization }\end{array}$ & $\begin{array}{l}\text { Penetration Depth } \\
\text { (confocal laser } \\
\text { scanning microscope) }\end{array}$ \\
\hline $\begin{array}{l}\text { Paris et al. 2011 A } \\
\text { (Germeny) [64] }\end{array}$ & $\begin{array}{l}\text { DFG: PA } 1508 / 1-2, \text { as part } \\
\text { partially by DMG. }\end{array}$ & 16 & $\begin{array}{l}\text { Human } \\
\text { (molars) }\end{array}$ & Cavitated lesions & $\begin{array}{c}\text { Without } \\
\text { demineralization }\end{array}$ & $\begin{array}{c}\text { Penetration Depth } \\
\text { (Confocal laser scanning } \\
\text { microscopy CLSM) }\end{array}$ \\
\hline $\begin{array}{l}\text { Taher et al. 2012 } \\
\text { (Saudi Arabia) [47] }\end{array}$ & No & 10 & $\begin{array}{c}\text { Human } \\
\text { (premolars) }\end{array}$ & $\begin{array}{l}\text { Cracks, restorations, or } \\
\text { developmental lesions }\end{array}$ & $\begin{array}{c}\text { Without } \\
\text { demineralization }\end{array}$ & $\begin{array}{l}\text { Roughness; Microhardness } \\
\text { (microscope with } 200 \\
\text { magnification and } \\
\text { application of applying a } \\
\text { load of } 300 \text { g; profilometer) }\end{array}$ \\
\hline $\begin{array}{l}\text { Torres et al. } 2012 \\
\quad \text { (Brazil) [48] }\end{array}$ & NR & 15 & $\begin{array}{l}\text { Bovine } \\
\text { (incisors) }\end{array}$ & $\begin{array}{l}\text { Damaged or not } \\
\text { intact enamel }\end{array}$ & $\mathrm{pH}: 5$ for $16 \mathrm{~h}$ & $\begin{array}{l}\text { Microhardness } \\
\text { (microhardness tester fitted } \\
\text { with a 50-g load) }\end{array}$ \\
\hline $\begin{array}{l}\text { Attin et al. 2012 } \\
\text { (Switzerland) [32] }\end{array}$ & $\begin{array}{l}\text { Dentaurum, 3M ESPE, } \\
\text { and DMG }\end{array}$ & 12 & $\begin{array}{l}\text { Bovine } \\
\text { (incisors) }\end{array}$ & NR & pH: NR for $504 \mathrm{~h}$ & $\begin{array}{c}\text { Shear Bond Strength } \\
\text { (universal testing machine) }\end{array}$ \\
\hline $\begin{array}{l}\text { Veli et al. } 2014 \\
\text { (Turkey) [72] }\end{array}$ & ( & 20 & $\begin{array}{c}\text { Human } \\
\text { (premolars) }\end{array}$ & $\begin{array}{l}\text { Caries, hypoplastic } \\
\text { areas, restorations, } \\
\text { and surface } \\
\text { abnormalities }\end{array}$ & $\mathrm{pH}: 4.8$ for $504 \mathrm{~h}$ & $\begin{array}{c}\text { Shear Bond Strength } \\
\text { (universal testing machine) }\end{array}$ \\
\hline $\begin{array}{l}\text { Ekizer et al. } 2012 \\
\quad \text { (Turkey) [70] }\end{array}$ & No & 20 & $\begin{array}{c}\text { Human } \\
\text { (premolars) }\end{array}$ & $\begin{array}{l}\text { Hypoplastic spots, } \\
\text { cracks, or gross } \\
\text { irregularities }\end{array}$ & $\mathrm{pH}: 4.3$ for $6 \mathrm{~h}$ & $\begin{array}{l}\text { Shear Bond Strength } \\
\text { (universal testing machine) }\end{array}$ \\
\hline $\begin{array}{l}\text { Paris et al. } 2013 \\
\text { (Germany)[43] }\end{array}$ & $\begin{array}{c}\text { DFG: PA1508/1-2. HML } \\
\text { and SP and royalties from } \\
\text { DMG, Hamburg. }\end{array}$ & 12 & $\begin{array}{l}\text { Bovine } \\
\text { (incisors) }\end{array}$ & NR & pH: 4.95 for $1200 \mathrm{~h}$ & $\begin{array}{c}\text { Microhardness } \\
\text { (Vickers hardness with a } \\
\text { force }(\mathrm{F}) \text { of } 0.981 \mathrm{~N} \text { for } 10 \mathrm{~s} \text { ) }\end{array}$ \\
\hline $\begin{array}{l}\text { Paris et al. } 2013 \\
\text { (Germany)[61] }\end{array}$ & DFG: PA 1508/1-1 & 15 & $\begin{array}{l}\text { Human } \\
\text { (molars and } \\
\text { premolars) }\end{array}$ & Cavitated caries & $\begin{array}{c}\text { Without } \\
\text { demineralization }\end{array}$ & $\begin{array}{c}\text { Penetration Depth } \\
\text { (confocal laser scanning) }\end{array}$ \\
\hline $\begin{array}{l}\text { Mohammed et al. } \\
2014 \text { (Iraq) [76] }\end{array}$ & NR & 56 & $\begin{array}{l}\text { Human } \\
\text { (premolars) }\end{array}$ & NR & $\mathrm{pH}: 4.5$ for $120 \mathrm{~h}$ & $\begin{array}{l}\text { Roughness } \\
\text { (profilometer) }\end{array}$ \\
\hline $\begin{array}{l}\text { Paris et al. } 2014 \\
\text { (Germany) [63] }\end{array}$ & DFG: PA 1508/1-3 & 9 & $\begin{array}{l}\text { Human } \\
\text { (molars and } \\
\text { premolars) }\end{array}$ & ICDAS codes $0,1,2$ & $\begin{array}{c}\text { Without } \\
\text { demineralization }\end{array}$ & $\begin{array}{l}\text { Penetration Depth } \\
\text { (dual fluorescence } \\
\text { confocal microscopy) }\end{array}$ \\
\hline $\begin{array}{l}\text { Lausch et al. 2014 } \\
\text { (Germany) [57] }\end{array}$ & $\begin{array}{l}\text { The Charité } \\
\text { Universitätsmedizin Berlin } \\
\text { and DMG }\end{array}$ & 17 & $\begin{array}{l}\text { Human } \\
\text { (molars and } \\
\text { premolars) }\end{array}$ & $\begin{array}{l}\text { Without active or } \\
\text { cavitated WSL }\end{array}$ & $\begin{array}{c}\text { Without } \\
\text { demineralization }\end{array}$ & $\begin{array}{l}\text { Penetration Depth } \\
\text { (confocal laser scanning) }\end{array}$ \\
\hline $\begin{array}{l}\text { Gelani et al. } 2014 \\
\text { (USA) [56] }\end{array}$ & 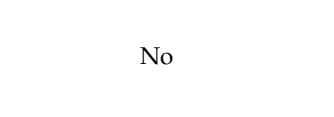 & 42 & $\begin{array}{l}\text { Bovine } \\
\text { (incisors) }\end{array}$ & $\begin{array}{l}\text { WSP, cracks, or any } \\
\text { other defect }\end{array}$ & $\mathrm{pH}: 5$ for $24 \mathrm{~h}$ & $\begin{array}{c}\text { Penetration Depth } \\
\text { (Confocal Laser Scanning } \\
\text { Microscopy and Transverse } \\
\text { Microradiography) }\end{array}$ \\
\hline $\begin{array}{l}\text { Dilber et al. 2014 } \\
\text { (Turkey) [69] }\end{array}$ & NR & 15 & $\begin{array}{c}\text { Human } \\
\text { (mandibular lateral } \\
\text { teeth) }\end{array}$ & $\begin{array}{l}\text { Hypoplastic areas, } \\
\text { cracks, or } \\
\text { gross irregularities } \\
\text { in enamel }\end{array}$ & ph: 4.3 for $6 \mathrm{~h}$ & $\begin{array}{l}\text { Shear Bond Strength } \\
\text { (Universal testing machine) }\end{array}$ \\
\hline $\begin{array}{l}\text { Montasser et al. } \\
2015 \text { (Egypt) [41] }\end{array}$ & No & 10 & $\begin{array}{l}\text { Human } \\
\text { (NR) }\end{array}$ & NR & pH: 4.4 for $504 \mathrm{~h}$ & $\begin{array}{l}\text { Microhardness } \\
\text { (Vickers diamond indenter } \\
\text { load of } 200 \mathrm{~g} \text { ) }\end{array}$ \\
\hline $\begin{array}{l}\text { Arslan et al. } 2015 \\
\quad \text { (Turkey) [31] }\end{array}$ & NR & 15 & $\begin{array}{c}\text { Human } \\
\text { (central incisors) }\end{array}$ & NR & $\mathrm{pH}: 4.5$ for $6 \mathrm{~h}$ & $\begin{array}{l}\text { Roughness; Microhardness } \\
\text { (profilometer; Vickers } \\
\text { hardness tester with } \\
2 \text { N load) }\end{array}$ \\
\hline
\end{tabular}


Table 1. Cont.

\begin{tabular}{|c|c|c|c|c|c|c|}
\hline Study & Funding & $n$ & Specimen Origin & $\begin{array}{l}\text { Exclusion } \\
\text { CRITERIA }\end{array}$ & $\begin{array}{l}\text { WSLs Preparation } \\
\text { (pH for hours) }\end{array}$ & Outcome Reported \\
\hline $\begin{array}{c}\text { Min et al. } 2015 \\
\text { (South Korea) [60] }\end{array}$ & $\begin{array}{l}\text { Basic Science Research } \\
\text { Program through the } \\
\text { National Research } \\
\text { Foundation of Korea } \\
\text { (2013R1A1A2062505) }\end{array}$ & 20 & $\begin{array}{l}\text { Bovine } \\
\text { (permanent anterior } \\
\text { teeth) }\end{array}$ & NR & $\mathrm{pH}: 4.8$ for $960 \mathrm{~h}$ & $\begin{array}{l}\text { Penetration Depth } \\
\text { (Optical coherence } \\
\text { tomography Confocal laser } \\
\text { scanning microscopy) }\end{array}$ \\
\hline $\begin{array}{l}\text { Vianna et al. } 2015 \\
\quad \text { (Brazil) [73] }\end{array}$ & No & 15 & $\begin{array}{l}\text { Bovine } \\
\text { (incisors) }\end{array}$ & NR & $\mathrm{pH}: 5$ for $56 \mathrm{~h}$ & $\begin{array}{l}\text { Shear Bond Strength } \\
\text { (universal testing machine) }\end{array}$ \\
\hline $\begin{array}{l}\text { Gurdogan et al. } \\
2016 \text { (Turkey) [38] }\end{array}$ & No & 20 & $\begin{array}{l}\text { Bovine } \\
\text { (incisors) }\end{array}$ & NR & $\mathrm{pH}: 4$ for $2 \mathrm{~h}$ & $\begin{array}{l}\text { Microhardness } \\
\text { (Vickers Hardness tester } \\
\text { with } 100 \text { gr force) }\end{array}$ \\
\hline $\begin{array}{l}\text { Abdel-Hakim et al. } \\
2016 \text { (Egypt) [28] }\end{array}$ & NR & 6 & $\begin{array}{l}\text { Human } \\
\text { (molars) }\end{array}$ & $\begin{array}{c}\text { Caries, } \\
\text { hypocalcifications, } \\
\text { or restorations }\end{array}$ & $\mathrm{pH}: 4.4$ for $480 \mathrm{~h}$ & $\begin{array}{c}\text { Microhardness } \\
\text { (Vickers michrohardness } \\
\text { testing with } 200 \text { gm load) } \\
\text { Microhardness: }\end{array}$ \\
\hline $\begin{array}{l}\text { El-zankalouny et al. } \\
2016 \text { (Egypt) [46] }\end{array}$ & No & 7 & $\begin{array}{c}\text { Human } \\
\text { (premolars) }\end{array}$ & $\begin{array}{l}\text { Cracks, caries, or } \\
\text { restorations }\end{array}$ & $\mathrm{pH}: 4.4$ for $96 \mathrm{~h}$ & $\begin{array}{c}\text { Penetration Depth } \\
\text { (Vickers tester with f } 50 \mathrm{~g} ; \\
\text { stereomicroscope) }\end{array}$ \\
\hline $\begin{array}{l}\text { Abdellatif et al. } \\
2016 \text { (Egypt) [29] }\end{array}$ & NR & 11 & $\begin{array}{c}\text { Human } \\
\text { (anterior teeth) }\end{array}$ & NR & pH: 4.8 for $720 \mathrm{~h}$ & $\begin{array}{c}\text { Microhardness } \\
\text { (Vicker's microhardness } \\
\text { test with load of } 200 \mathrm{~g} \text { ) }\end{array}$ \\
\hline $\begin{array}{l}\text { Baka et al. } 2016 \\
\text { (Turkey) [52] }\end{array}$ & NR & 20 & $\begin{array}{c}\text { Human } \\
\text { (premolars) }\end{array}$ & $\begin{array}{l}\text { Hypoplastic areas, } \\
\text { cracks, restorations, } \\
\text { or gross } \\
\text { irregularities }\end{array}$ & pH: 4.8 for $504 \mathrm{~h}$ & $\begin{array}{l}\text { Roughness; Shear } \\
\text { Bond Strengths } \\
\text { (profilometer; a universal } \\
\text { testing machine) }\end{array}$ \\
\hline $\begin{array}{l}\text { Neto et al. } 2016 \\
\text { (Brazil) [30] }\end{array}$ & $\begin{array}{c}\text { CAPES, Funcap, and } \\
\text { CNPq (Brazilian agencies). } \\
\text { Proiect PON 254/Ric }\end{array}$ & 10 & $\begin{array}{l}\text { Human } \\
\text { (molars) }\end{array}$ & NR & $\mathrm{pH}: 4.9$ for $16 \mathrm{~h}$ & $\begin{array}{c}\text { Microhardness } \\
\text { (Knoop microhardness) }\end{array}$ \\
\hline $\begin{array}{l}\text { Horuztepe et al. } \\
2017 \text { (Turkey) [39] }\end{array}$ & 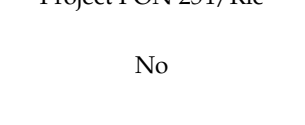 & 45 & $\begin{array}{l}\text { Bovine } \\
\text { (incisors) }\end{array}$ & $\begin{array}{l}\text { Cracks or other } \\
\text { surface defects }\end{array}$ & pH: 4.95 for $672 \mathrm{~h}$ & $\begin{array}{c}\text { Microhardness } \\
\text { (microindentation } \\
\text { hardness tester with a } \\
\text { 50-g load) }\end{array}$ \\
\hline $\begin{array}{l}\text { Mandava et al. } 2017 \\
\text { (India) [40] }\end{array}$ & No & 20 & $\begin{array}{l}\text { Human } \\
\text { (maxillary central } \\
\text { incisors) }\end{array}$ & $\begin{array}{l}\text { Presence of cracks } \\
\text { and defects }\end{array}$ & $\mathrm{pH}: 4.4$ for $96 \mathrm{~h}$ & $\begin{array}{c}\text { Microhardness; } \\
\text { Penetration Depth } \\
\text { (Vicker's microhardness } \\
\text { tester with a } 300 \text { g load; } \\
\text { confocal laser } \\
\text { fluorescence microscope) }\end{array}$ \\
\hline $\begin{array}{l}\text { Aziznezhad et al. } \\
2017 \text { (Iran) [34] }\end{array}$ & Babol University grant & 10 & $\begin{array}{c}\text { Human } \\
\text { (premolars) }\end{array}$ & $\begin{array}{l}\text { Not intact and time } \\
\text { of extraction more } \\
\text { than } 3 \text { months }\end{array}$ & $\mathrm{pH}: 4.5$ for $6 \mathrm{~h}$ & $\begin{array}{l}\text { Microhardness } \\
\text { (Vickers device with } \\
500 \mathrm{~g} \text { load) }\end{array}$ \\
\hline $\begin{array}{l}\text { Prajapati et al. } 2017 \\
\text { (India) [44] }\end{array}$ & No & 10 & $\begin{array}{c}\text { Human } \\
\text { (premolars) }\end{array}$ & $\begin{array}{c}\text { Teeth with } \\
\text { hypoplasia or } \\
\text { incipient carious } \\
\text { lesions/WSL }\end{array}$ & $\mathrm{pH}: 4.4$ for $504 \mathrm{~h}$ & $\begin{array}{l}\text { Microhardness } \\
\text { (Vickers microhardness } \\
\text { tester with } 100 \mathrm{~g} \text { load) }\end{array}$ \\
\hline $\begin{array}{l}\text { Sava-Rosianu et al. } \\
2017 \text { (Romania) [65] }\end{array}$ & $\begin{array}{l}\text { Project for young } \\
\text { researchers-Programme } \\
\text { II-C3-TC-2015 }\end{array}$ & 60 & $\begin{array}{l}\text { Human } \\
\text { (premolar) }\end{array}$ & 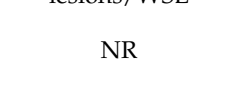 & NR & $\begin{array}{l}\text { Penetration Depth } \\
\text { (Confocal Laser } \\
\text { Scanning Microscopy) }\end{array}$ \\
\hline $\begin{array}{l}\text { Attia et al. } 2018 \\
\text { (Egypt) [77] }\end{array}$ & (1) & 20 & $\begin{array}{l}\text { Bovine } \\
(\mathrm{NR})\end{array}$ & $\begin{array}{l}\text { Cracks or defects in } \\
\text { the surface }\end{array}$ & $\mathrm{pH}: 5$ for $24 \mathrm{~h}$ & $\begin{array}{c}\text { Microhardness } \\
\text { (micro-indentation } \\
\text { hardness tester (with a } \\
50 \text {-g load) }\end{array}$ \\
\hline $\begin{array}{l}\text { Nabil et al. } 2018 \\
\quad \text { (Egypt) [27] }\end{array}$ & NR & 15 & $\begin{array}{c}\text { Human } \\
\text { (anterior teeth) }\end{array}$ & $\begin{array}{l}\text { Cracks and any } \\
\text { developmental } \\
\text { defects }\end{array}$ & $\mathrm{pH}$ : NR for $1 \mathrm{~h}$ & $\begin{array}{l}\text { Roughness; Microhardness } \\
\text { (profilometer; Vickers } \\
\text { Tester with load of } 200 \mathrm{~g} \text { ) }\end{array}$ \\
\hline $\begin{array}{l}\text { Enan et al. } 2018 \\
\text { (Egypt) [37] }\end{array}$ & NR & 10 & $\begin{array}{l}\text { Human } \\
\text { (bicuspid) }\end{array}$ & Cracks and defects & $\mathrm{pH}: 4.95$ for $160 \mathrm{~h}$ & $\begin{array}{l}\text { Roughness; Microhardness } \\
\text { (profilometer; universal } \\
\text { testing machine) }\end{array}$ \\
\hline $\begin{array}{l}\text { Khalid et al. } 2018 \\
\text { (Indonesia) [54] }\end{array}$ & University of Indonesia & 10 & $\begin{array}{c}\text { Human } \\
\text { (premolars) }\end{array}$ & $\begin{array}{l}\text { Enamel surface that } \\
\text { was attached } \\
\text { orthodontic } \\
\text { appliance; WSL, } \\
\text { defects on the } \\
\text { buccal side of } \\
\text { enamel; restorations }\end{array}$ & $\begin{array}{c}\text { Without } \\
\text { demineralization }\end{array}$ & $\begin{array}{l}\text { Roughness } \\
\text { (profilometer) }\end{array}$ \\
\hline $\begin{array}{l}\text { Yazkan et al. } 2018 \\
\text { (Turkey) [49] }\end{array}$ & $\begin{array}{l}\text { Suleyman Demirel } \\
\text { University Scientific } \\
\text { Research Projects } \\
\text { Foundation (2969-D-11) }\end{array}$ & 16 & $\begin{array}{l}\text { Bovine } \\
\text { (incisors) }\end{array}$ & $\begin{array}{l}\text { Caries, fracture, or } \\
\text { other defects }\end{array}$ & $\mathrm{pH}: 5$ for $240 \mathrm{~h}$ & $\begin{array}{l}\text { Roughness; Microhardness } \\
\text { (profilometer; Vickers } \\
\text { indenter, with load of } \\
200 \mathrm{~g} \text { ) }\end{array}$ \\
\hline $\begin{array}{l}\text { Askar et al. } 2018 \\
\text { (Germany) [55] }\end{array}$ & $\begin{array}{c}\text { Deutsche } \\
\text { Forschungsgemeinschaft } \\
\text { (DFG; PA 1508/1-3), } \\
\text { and DMG }\end{array}$ & 15 & $\underset{\text { (NR) }}{\text { Human }}$ & $\begin{array}{l}\text { Active proximal } \\
\text { lesions with } \\
\text { ICDAS-2, } 3 \text { and } 5\end{array}$ & $\begin{array}{c}\text { Without } \\
\text { demineralization }\end{array}$ & $\begin{array}{l}\text { Penetration Depth } \\
\text { (confocal microscopy) }\end{array}$ \\
\hline $\begin{array}{l}\text { Aswani et al. } 2019 \\
\quad \text { (India) [51] }\end{array}$ & No & 10 & $\begin{array}{l}\text { Human } \\
\text { (anterior teeth) }\end{array}$ & NR & $\mathrm{pH}: 4.4$ for $144 \mathrm{~h}$ & $\begin{array}{l}\text { Roughness } \\
\text { (profilometer) }\end{array}$ \\
\hline $\begin{array}{l}\text { Enan et al. } 2019 \\
\text { (Egypt) [53] }\end{array}$ & No & 30 & $\begin{array}{l}\text { Human } \\
\text { (premolars) }\end{array}$ & NR & $\mathrm{pH}: 4.95$ for $160 \mathrm{~h}$ & $\begin{array}{l}\text { Roughness } \\
\text { (profilometer) }\end{array}$ \\
\hline
\end{tabular}


Table 1. Cont.

\begin{tabular}{|c|c|c|c|c|c|c|}
\hline Study & Funding & $n$ & Specimen Origin & $\begin{array}{l}\text { Exclusion } \\
\text { CRITERIA }\end{array}$ & $\begin{array}{l}\text { WSLs Preparation } \\
\text { (pH for hours) }\end{array}$ & Outcome Reported \\
\hline $\begin{array}{l}\text { Arora et al. } 2019 \\
\text { (India) [50] }\end{array}$ & 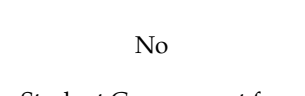 & 30 & $\begin{array}{c}\text { Bovine } \\
\text { (premolars) }\end{array}$ & Caries & $\mathrm{pH}: 4.5$ for $96 \mathrm{~h}$ & $\begin{array}{c}\text { Roughness; } \\
\text { Penetration Depth } \\
\text { (optical profilometer) }\end{array}$ \\
\hline $\begin{array}{l}\text { Theodory et al. } 2019 \\
\text { (USA) [66] }\end{array}$ & $\begin{array}{c}\text { Student Government for } \\
\text { Graduate and Professional } \\
\text { Students at the University } \\
\text { of Iowa }\end{array}$ & 15 & $\begin{array}{l}\text { Human } \\
\text { (molars) }\end{array}$ & NR & $\mathrm{pH}: 4.3$ for $2160 \mathrm{~h}$ & $\begin{array}{c}\text { Penetration Depth } \\
\text { (Confocal Laser Scanning } \\
\text { Microscopy) }\end{array}$ \\
\hline $\begin{array}{l}\text { López et al. } 2019 \\
\text { (Brazil) [59] }\end{array}$ & 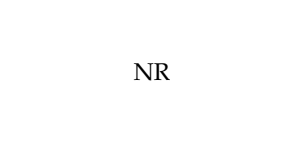 & 8 & $\underset{\text { (NR) }}{\text { Human }}$ & $\begin{array}{c}\text { Cavity lesions, } \\
\text { white stains, cracks, } \\
\text { or structural } \\
\text { alterations and } \\
\text { restorations }\end{array}$ & $\mathrm{pH}: 5$ for $0.5 \mathrm{~h}$ & $\begin{array}{l}\text { Penetration Depth } \\
\text { (Confocal Laser } \\
\text { Scanning Microscopy) }\end{array}$ \\
\hline $\begin{array}{l}\text { Gulec et al. } 2019 \\
\text { (Turkey) [71] }\end{array}$ & NR & 20 & $\begin{array}{c}\text { Human } \\
\text { (premolars) }\end{array}$ & $\begin{array}{l}\text { Caries, attrition, } \\
\text { fracture, restoration, } \\
\text { congenital or } \\
\text { surface anomalies, } \\
\text { or surface }\end{array}$ & $\mathrm{pH}: 4.5$ for $22 \mathrm{~h}$ & $\begin{array}{c}\text { Shear Bond Strength } \\
\text { (universal testing machine) }\end{array}$ \\
\hline $\begin{array}{l}\text { Borges et al. } 2019 \\
\text { (Brazil) [68] }\end{array}$ & $\begin{array}{c}\text { FAPESP(2010/16878-7, } \\
2010 / 17757-9)\end{array}$ & 30 & $\begin{array}{l}\text { Bovine } \\
\text { (incisors) }\end{array}$ & 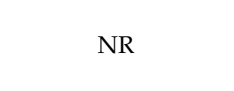 & $\mathrm{pH}: 5$ for $16 \mathrm{~h}$ & $\begin{array}{l}\text { Shear Bond Strength } \\
\text { (Scanning electron } \\
\text { microscopy (SEM)) }\end{array}$ \\
\hline $\begin{array}{l}\text { Ayad et al. } 2020 \\
\text { (Egypt) [33] }\end{array}$ & NR & 7 & $\begin{array}{l}\text { Bovine } \\
\text { (anteriors) }\end{array}$ & NR & $\mathrm{pH}: 4.4$ for $96 \mathrm{~h}$ & $\begin{array}{l}\text { Microhardness } \\
\text { (Vickers indenter, with a } \\
\text { static load of } 200 \mathrm{~g} \text { ) }\end{array}$ \\
\hline $\begin{array}{l}\text { Behrouzi P et al. } \\
2020 \text { (Iran) [35] }\end{array}$ & No & 15 & $\begin{array}{c}\text { Human } \\
\text { (maxillary central } \\
\text { incisors) }\end{array}$ & $\begin{array}{l}\text { Cracks, caries, or } \\
\text { mineralization } \\
\text { defects }\end{array}$ & $\mathrm{pH}: 4.5$ for $96 \mathrm{~h}$ & $\begin{array}{c}\text { Microhardness } \\
\text { (Vickers hardness tester } \\
\text { with } 50 \mathrm{~kg} \text { load) }\end{array}$ \\
\hline $\begin{array}{l}\text { El Meligy, 2020 } \\
\text { (Saudi Arabia) [36] }\end{array}$ & No & 27 & $\begin{array}{c}\text { Human } \\
\text { (premolars) }\end{array}$ & ICDAS 1 and 2 & $\mathrm{pH}: 4.5$ for $399 \mathrm{~h}$ & $\begin{array}{c}\text { Microhardness } \\
\text { (transversal Vickers } \\
\text { hardness with a force of } \\
0.891 \mathrm{~N})\end{array}$ \\
\hline $\begin{array}{l}\text { Wang et al. } 2020 \\
\text { (Brazil) [67] }\end{array}$ & $\begin{array}{c}\text { FAPESP, } 2012 / 13160-3, \\
\text { \#2012/18579-2 and } \\
2013 / 23310-5) \\
\text { CAPES-Brasil }\end{array}$ & 13 & $\begin{array}{l}\text { Bovine } \\
\text { (incisors) }\end{array}$ & NR & $\mathrm{pH}: 4.7$ for $168 \mathrm{~h}$ & $\begin{array}{l}\text { Penetration Depth } \\
\text { (confocal laser } \\
\text { scanning microscopy) }\end{array}$ \\
\hline
\end{tabular}

CAPES-Coordenação de Aperfeiçoamento de Pessoal de Nível Superior; CLSM-Confocal Laser Scanning Microscope; CNPq-Conselho Nacional de Desenvolvimento Científico e Tecnológico; DFG—Deutsche Forschungsgemeinschaft; FAPESP-Fundação de Amparo à Pesquisa do Estado de São Paulo; FUNCAP—Fundação Cearense de Apoio ao Desenvolvimento Científico e Tecnológico; H-Hours; HML—Hendrik Meyer-Lueckel; ICDAS—International Caries Detection and Assessment System; NR—Not reported; WSL—white spots lesions; S.P-Sebastian Paris.

\subsection{Methodological Quality of the Included Studies}

Methodological appraisal of the included in-vitro studies using the Joanna Briggs Institute Clinical Appraisal Checklist for Experimental Studies tool is presented in Figure 2 and is detailed in Table S3. The assessment varied from 7 to 10 (one article with score 7, twenty-two with score 8 , twenty-two with score 9 and three with score 10). All included studies showed a clear objective $(n=48,100 \%)$ and treated the specimens from the control and experimental group using the same protocol $(n=48,100 \%)$. Furthermore, all articles used an appropriate statistical analysis $(n=48,100 \%)$ and presented reliable outcomes ( $n=48,100 \%)$. The majority carefully described the preparation protocol $(n=44,91.7 \%)$, and the experimental protocol to characterize the several steps and materials applied $(n=39,81.3 \%)$. On the opposite, most articles failed on sample size justification $(n=41$, $85.4 \%)$, in the random assignment of treatment groups $(n=15,31.3 \%)$, and only one study reported blindness regarding treatment allocation $(n=1,2.1 \%)$.

\subsection{Clinical Measures}

An a priori sensitivity analysis was performed to compare whether both ROM and SMD approaches yielded results in terms of significance and heterogeneity results (Table S4). Overall, ROM and SMD meta-analyses presented similar significance and heterogeneity degrees, therefore supporting the use of a ROM meta-analytical approach (Table S4). 


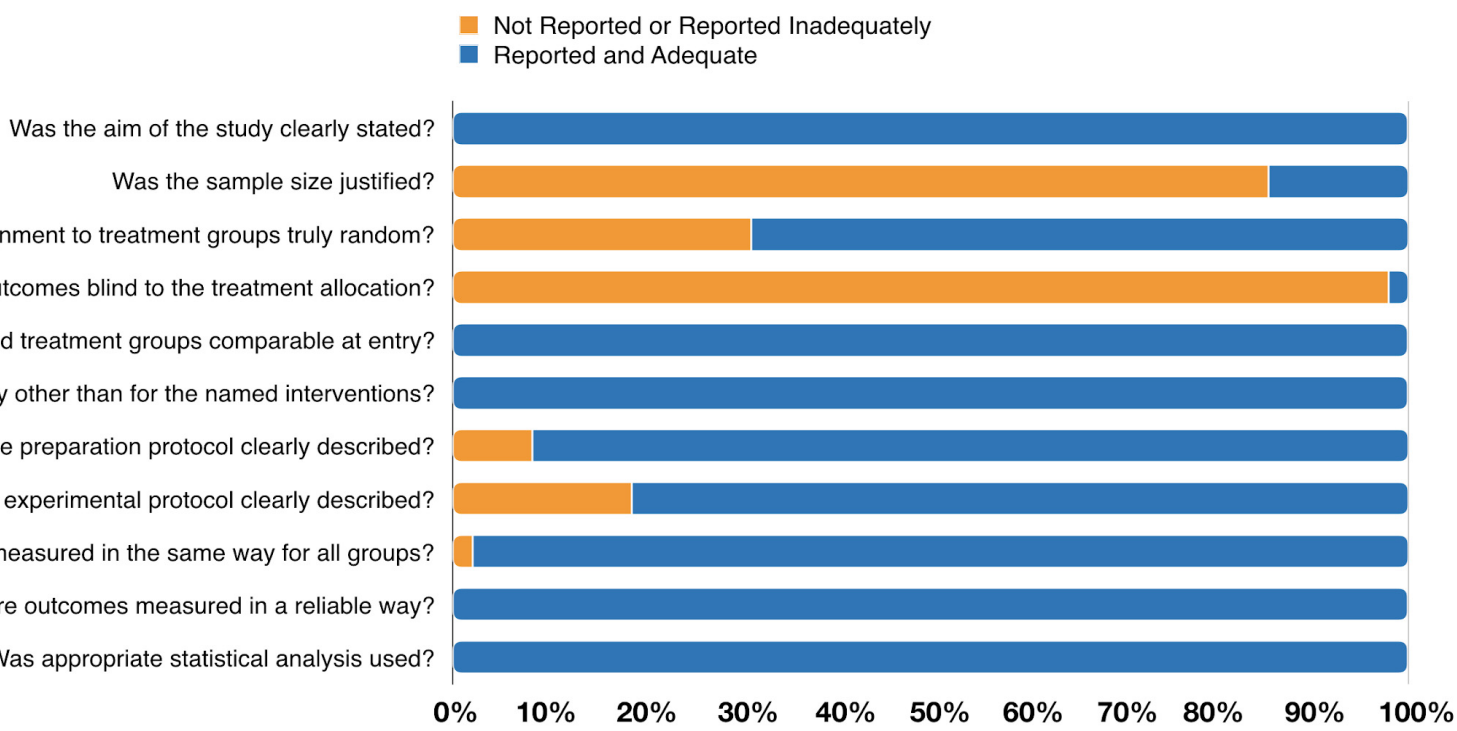

Figure 2. Assessment of the risk of bias in the included studies according to the percentage of the scores attributed to each evaluated study.

\subsubsection{Enamel Surface Roughness}

Surface roughness before and after resin application was analyzed in both sound enamel and in WSLs, only in human teeth samples (Tables 2 and 3). It is possible to attest that resin infiltration decreases the 35\% (ROM $=0.65,95 \%$ CI: $0.49 ; 0.85, p<0.0021)$ the surface roughness in sound enamel and $54 \%$ in WSLs (ROM $=0.46,95 \%$ CI: 0.29; 0.74, 0.0012) (Table 2, Figures S1 and S2). In both estimates' heterogeneity was considered high $\left(\mathrm{I}^{2}=98.2 \%\right.$ and $\mathrm{I}^{2}=98.5 \%$, respectively) (Table 2$)$. Furthermore, multivariate sensitivity analysis demonstrated $\mathrm{pH}$ and time of exposure to the demineralizing agent had an important impact on surface roughness ( $\mathrm{ROM}=-1.37,95 \% \mathrm{CI}:-2.32 ;-0.42, p<0.005$; $\mathrm{ROM}=0.01,95 \%$ CI: 0.00; 0.01, $p<0.001$, respectively) (Table 4).

Table 2. Sound enamel and white spot lesions according to surface roughness, enamel microhardness, bond strength and penetration depth.

\begin{tabular}{|c|c|c|c|c|c|c|}
\hline Variable & $n$ Studies & ROM & $95 \% \mathrm{CI}$ & $p$-Value & $I^{2}(\%)$ & Egger Test \\
\hline \multicolumn{7}{|c|}{ Surface roughness } \\
\hline Sound Enamel & 5 & 0.65 & $0.49 ; 0.85$ & 0.0021 & 98.2 & - \\
\hline WSL & 8 & 0.46 & $0.29 ; 0.74$ & 0.0012 & 98.5 & - \\
\hline \multicolumn{7}{|c|}{ Enamel microhardness } \\
\hline Sound Enamel & 14 & 0.76 & $0.73 ; 0.8$ & $<0.0001$ & 99.1 & 0.8893 \\
\hline WSL & 23 & 1.68 & $1.51 ; 1.86$ & $<0.0001$ & 99.8 & 0.1352 \\
\hline \multicolumn{7}{|c|}{ Shear Bond strength } \\
\hline Sound Enamel & 6 & 0.75 & $0.60 ; 0.95$ & $<0.0001$ & 96.9 & - \\
\hline WSL & 8 & 1.89 & $1.28 ; 2.79$ & $<0.0001$ & 99.8 & - \\
\hline \multicolumn{7}{|c|}{ Penetration depth } \\
\hline Sound Enamel & 15 & 65.39 & $56.11 ; 74.66$ & 0.01 & 100.0 & 0.4712 \\
\hline
\end{tabular}

CI—Confidence Interval; N—number; ROM—Ratio of Means; WSL—White Spot Lesions. Bold-face denotes significance.

Table 3. Sensitivity analysis of type of samples using meta-regressions.

\begin{tabular}{|c|c|c|c|c|c|}
\hline Specimen Origin & $n$ & B & $95 \% \mathrm{CI}$ & $I^{2}(\%)$ & $p$-Value \\
\hline \multicolumn{6}{|c|}{ Surface Roughness of Sound Enamel } \\
\hline Human & 4 & 0.65 & $0.49 ; 0.85$ & 98.8 & - \\
\hline Bovine & 0 & - & - & - & - \\
\hline
\end{tabular}


Table 3. Cont.

\begin{tabular}{|c|c|c|c|c|c|}
\hline Specimen Origin & $n$ & B & $95 \% \mathrm{CI}$ & $I^{2}(\%)$ & $p$-Value \\
\hline \multicolumn{6}{|c|}{ Surface Roughness of WSL } \\
\hline Human & 8 & 0.46 & $0.29 ; 0.74$ & 98.5 & \\
\hline Bovine & 0 & - & - & - & \\
\hline \multicolumn{6}{|c|}{ Enamel Microhardness of Sound Enamel } \\
\hline Human & 8 & 0.58 & $0.46 ; 0.72$ & 99.0 & \multirow[b]{2}{*}{0.0188} \\
\hline Bovine & 6 & 0.80 & $0.69 ; 0.92$ & 99.3 & \\
\hline \multicolumn{6}{|c|}{ Enamel Microhardness of WSL } \\
\hline Human & 15 & 1.59 & $1.29 ; 1.96$ & 99.5 & \multirow[b]{2}{*}{0.1375} \\
\hline Bovine & 8 & 1.96 & $1.64 ; 2.34$ & 99.4 & \\
\hline \multicolumn{6}{|c|}{ Shear Bond Strength of Sound Enamel } \\
\hline Human & 4 & 0.75 & $0.57 ; 0.99$ & 97.2 & \multirow{2}{*}{0.9958} \\
\hline Bovine & 2 & 0.75 & $0.41 ; 1.37$ & 97.7 & \\
\hline \multicolumn{6}{|c|}{ Shear Bond Strength of WSL } \\
\hline Human & 5 & 1.74 & 1.14: 2.65 & 98.7 & \multirow{2}{*}{0.6221} \\
\hline Bovine & 3 & 2.20 & $0.93: 5.29$ & 98.8 & \\
\hline \multicolumn{6}{|c|}{ Penetration Depth } \\
\hline Human & 20 & 63.65 & $52.21 ; 75.09$ & 99.3 & \multirow[b]{2}{*}{0.5589} \\
\hline Bovine & 6 & 71.22 & $48.57 ; 93.87$ & 99.8 & \\
\hline ICDAS & 16 & 62.37 & $46.12 ; 78.61$ & 99.2 & \multirow{2}{*}{0.4500} \\
\hline WSLs & 10 & 70.29 & $57.68 ; 82.90$ & 99.7 & \\
\hline $\begin{array}{l}\text { Application of infiltrate for less } \\
\text { than } 1 \mathrm{~min}\end{array}$ & 4 & 49.17 & $33.36 ; 64.97$ & 94.1 & \multirow{3}{*}{0.0270} \\
\hline Application of infiltrate for $3 \mathrm{~min}$ & 18 & 65.36 & $55.77 ; 74.96$ & 99.4 & \\
\hline Application of infiltrate for $5 \mathrm{~min}$ & 4 & 81.45 & $63.88 ; 99.01$ & 96.5 & \\
\hline
\end{tabular}

CI-Confidence Interval; N-number; ROM-Ratio of Means; WSL-White Spot Lesions.

Table 4. Sensitivity analysis of $\mathrm{pH}$ and demineralization time using meta-regressions.

\begin{tabular}{|c|c|c|c|c|c|c|}
\hline \multicolumn{4}{|c|}{ Univariate } & \multicolumn{3}{|c|}{ Multivariate } \\
\hline Charateristics & B & $95 \% \mathrm{CI}$ & $p$-Value & B & $95 \%$ CI & $p$-Value \\
\hline \multicolumn{7}{|c|}{ Surface roughness of WSLs } \\
\hline $\mathrm{pH}$ & -0.51 & $-2.96 ; 1.93$ & 0.6806 & -1.37 & $-2.32 ;-0.42$ & 0.00451 \\
\hline Demineralization time (hours) & 0.00 & $-0.00 ; 0.01$ & 1.8929 & 0.01 & $0.00 ; 0.01$ & $<0.0001$ \\
\hline \multicolumn{7}{|c|}{ Enamel microhardness of WSLs } \\
\hline $\mathrm{pH}$ & 0.42 & $-3.42 ; 0.67$ & 0.0641 & 0.42 & $-0.03 ; 0.00$ & 0.0647 \\
\hline Demineralization time (hours) & 0.00 & $-0.01 ; 0.00$ & 0.6230 & 0.00 & $-0.01 ; 0.00$ & 0.8729 \\
\hline \multicolumn{7}{|c|}{ Shear Bond Strength of WSLs } \\
\hline $\mathrm{pH}$ & -0.59 & $-6.98 ; 7.62$ & 0.9781 & -0.60 & $-2.02 ; 0.82$ & 0.4098 \\
\hline Demineralization time (hours) & 0.00 & $0.00 ; 0.01$ & 0.0015 & 0.00 & $0.00 ; 0.01$ & 0.0120 \\
\hline \multicolumn{7}{|c|}{ Penetration depth of WSLs } \\
\hline $\mathrm{pH}$ & -6.24 & $-54.77 ; 42.29$ & 0.8010 & -33.63 & $-0.88 ; 290.10$ & 0.0432 \\
\hline Demineralization time (hours) & 0.00 & $-0.02 ; 0.02$ & 0.9969 & 0.00 & $-0.01 ; 0.01$ & 0.8542 \\
\hline $\begin{array}{l}\text { Time of application of resin } \\
\text { infiltrate (minutes) }\end{array}$ & 7.68 & $1.17 ; 14.18$ & 0.0207 & 24.12 & $13.16 ; 35.07$ & $<0.0001$ \\
\hline
\end{tabular}

CI-Confidence Interval.

\subsubsection{Enamel Microhardness}

Our results showed that resin infiltration significantly reduced by $24 \%$, on average, the microhardness of sound enamel $(\mathrm{ROM}=0.76,95 \% \mathrm{CI}: 0.73 ; 0.80, p<0.001)$ and to increase by $68 \%$ the microhardness of enamel with WSLs (ROM $=1.68,95 \%$ CI: $1.51 ; 1.86, p<0.001)$ (Table 2, Figures S3 and S4). In both estimates, the heterogeneity was high $\left(\mathrm{I}^{2}=99.1 \%\right.$ and $\mathrm{I}^{2}=99.8 \%$, respectively), and there was no publication bias in both analyses $(0.8893$ and 0.1352, respectively) (Table 2, Figures S5 and S6). In particular, the enamel microhardness of sound enamel of human teeth was different compared to bovine teeth $(p<0.0188)$, showing that the enamel microhardness of sound enamel after resin application was lower in human teeth compared to bovine teeth $(\mathrm{ROM}=0.58,95 \% \mathrm{CI}: 0.46 ; 0.72$, and $\mathrm{ROM}=0.80$, 
95\% CI: $0.69 ; 0.92, p<0.0188)$. Although without any significant difference $(p=0.1375)$, the same pattern was found in human and bovine teeth with WSLs (ROM = 1.59, 95\% CI: 1.29; 1.96 and $1.96,95 \%$ CI: $1.64 ; 2.34$, respectively) (Table 3). Still, sensitivity analysis showed no differences based on $\mathrm{pH}$ and demineralization time, both using univariate and multivariate analysis $(p=0.0188)$ (Table 4$)$.

\subsubsection{Shear Bond Strength}

In what concerns shear bond strength, resin infiltration was estimated to reduce $25 \%$ the bond strength in sound enamel (ROM $=0.75,95 \% \mathrm{CI}: 0.60 ; 0.95, p<0.001)$ and to increase by $89 \%$ the bond strength in WSLs (ROM $=1.89,95 \% \mathrm{CI}: 1.28 ; 2.79, p<0.001)$ (Table 2, Figures S7 and S8). In both estimates, heterogeneity was high $\left(\mathrm{I}^{2}=96.9 \%\right.$ and $\mathrm{I}^{2}=99.8 \%$, respectively) (Table 2 ). In addition, there was no difference between human and bovine teeth in both analyses $(p=0.6221)$ (Table 3$)$, and only the demineralization time had an important impact on bond strength of teeth with WSLs, both in univariate and multivariate analyses (ROM $=0.00,95 \% \mathrm{CI}: 0.00 ; 0.01, p=0.0015$ and $\mathrm{ROM}=0.00$, 95\% CI: 0.00; 0.01, $p=0.0120$, respectively) (Table 4).

\subsubsection{Penetration Depth in Caries Lesions}

Regarding the penetration depth, only studies with enamel lesions were included. Considering the sound enamel the baseline value as zero, resin infiltration was estimated to penetrate $65.4 \%$ of overall lesion (MRAW $=65.4,95 \%$ CI: $56.11 ; 74.66, p=0.01, \mathrm{I}^{2}=100 \%$ ) (Table 2, Figure S9). In addition, the longer the application time, the greater the average penetration depth of the resin (Table 3, Figures S9 and S10).

\section{Discussion}

\subsection{Summary of the Main Results}

Overall, the present systematic review demonstrates that infiltrative resins effectively change the properties of both sound enamel and WSLs. In sound enamel, infiltrative resins reduced surface roughness, microhardness and shear bond strength. Regarding WSLs, infiltrative resins reduced enamel surface roughness, but increased its microhardness and shear bond strength. Furthermore, estimates point to an average penetration depth capacity of $65 \%$ by this type of resin.

\subsection{Quality of the Evidence and Potential Biases in the Review Process}

There are limitations inherent to the included studies. The protocols to create artificial WSLs differ in the $\mathrm{pH}$ of the demineralized agent used and etching time, and this could be a source of heterogeneity. Our sensitivity analyses via meta-regression confirmed that $\mathrm{pH}$ significantly influences surface roughness in WSLs and resin penetration depth after infiltration technique, and the etching time affected surface roughness and shear bond strength in WSLs. Thus, these protocol variations might affect the interaction with superficial crystals [78] and, therefore, might have contributed to the heterogeneity in the estimates. Additionally, our estimates included both studies on bovine and human teeth. Although this could undermine the consistency of the results, sensitivity analyses only showed an effect on microhardness in sound enamel specimens, and for the remaining analyses there was no significant impact. Nevertheless, future studies shall look for a harmonization of the protocol of WSL creation as well the specimen origin towards a consistent study methodology. In addition, most studies lacked an appropriate rationale for sample size calculation and group allocation of specimens, and these should be accounted for in future studies.

This systematic review also presents some strengths that are worth discussing. A strict protocol was followed with a guideline-based methodology and extensive scientific search. To the best of our knowledge, this is the first systematic review to demonstrate how much infiltrative resins can improve surface roughness, shear bond strength and penetration depth in permanent teeth with and without enamel lesions. Regarding microhardness, 
although one systematic review had estimated the impact of microhardness on WSLs and sound enamel [20], our approach explored for the first time the effect of $\mathrm{pH}$ of the demineralized agent used, etching time, and tooth origin through meta-regression sensitivity analysis on this enamel characteristic. In addition, we included 10 and 16 new studies on sound enamel and WSL, respectively (350\% and $229 \%$ of the total number of included studies [20], and more than 800 specimens (170\% of the total number of specimens) comparing to previous systematic review [20]. Furthermore, by using two meta-analytical approaches and the effort to detect and mitigate potential sources of heterogeneity, we are secure with the effect sizes across the included studies.

\subsection{Agreements and Disagreements with Other Reviews or Studies and Clinical Relevance}

Resin infiltration technique is a minimally invasive therapy to WSLs [78] with a preetching phase onto the lesion to improve penetration ability [28,77]. In addition, this penetrative role is enhanced by its methacrylate-based resin matrix containing BisGMA (bisphenol A diglycidil dimethacrylate) and TEGDMA (triethylene glycol dimethacrylate) $[43,49]$, which confer low viscosity to the resin [35].

Analyzing our results on surface roughness, both sound enamel and WSLs decreased the roughness after resin-infiltration application (35\% and $54 \%$, respectively), and these results have clinical importance. The oral cavity constantly undergoes a dynamic demineralization-remineralization cycle that promotes natural healing processes [79]. On the one hand, oral biofilm and dietary acids can contribute to create porous lesions on enamel, and, on the other hand, saliva, sealants, antibacterials, fluoride, and a controlled diet with less sugar and starchy foods promote a non-demineralizing environment [80]. Hence, infiltrative resins may play a role not only interventional but also preventive in the enamel roughness resulting throughout life.

Microhardness is a linear enamel characteristic based on the local calcium content [41], and this parameter can be used to assess the increase or reduction of percentage of enamel porosity [28]. Although resin infiltration might increase the microhardness, the establishment of the polymeric chain does not always happen in the entire lesion [81]. Therefore, the inability of a strong intermolecular bond plus the non-infiltration of the resin in the entire enamel lesion can prevent the full recovery of the enamel microhardness. Our results confirmed that the resin infiltration cannot return the microhardness of WSLs to that of sound enamel, although it may restore $68 \%$ of it. This result is in agreement with one systematic review that had shown a 3.66 mean difference increase compared with untreated samples [20]. Furthermore, our results fully comply with this previous work with a similar level of heterogeneity. Yet, as above mentioned, our results explored other characteristics that until today had not been in an evidence-based manner.

Shear bond strength concerns the amount of force required to break the adhesive/adherend interface connection [82], and our results showed this characteristic decreases $25 \%$ in sound enamel and increases $89 \%$ in WSLs. These results are consistent with the literature [52,70]. Firstly, enamel lesions have a degree of porosity with high permeability, allowing the infiltration of the resin. This ultimately results in micromechanical interdigitation strengthening, and therefore increases shear bond strength [52,70]. Secondly, the decrease of shear bond strength on sound enamel may be justified by the low quality of the enamel surface and lack of resin tags for mechanical interlocking [52].

The resin infiltration of WSLs with low viscosity resin results in a hybrid enamel with resin tags that impregnates the interprismatic enamel and reinforces the hard tissue $[60,83,84]$. Despite its qualities, not the whole portion of the lesion is filled with the resin $[40,65,66]$. Furthermore, increasing time of application of resin infiltration improves depth penetration $[59,63]$. The 'Washburn equation' describes the time-dependent as an important characteristic to advantage the viscosity, surface tension, and contact angle and allows the resin penetration into porous solids [68]. Comprehensively, our results highlight $65,35 \%$ of overall enamel lesions were filled with resin, and the longer the application time, 
the greater the average penetration depth. Our results are in fully agreement with the literature $[43,50,77]$.

The animal origin of the samples may explain the heterogeneity observed. Bovine teeth are often used in this type of studies, due to their similarities to human teeth [85]. Bovine teeth have a larger crystalline diameter, and their calcium distribution is more homogenous [86]. This species also has a lower fluoride concentration and increased porosity [87]. Nevertheless, the calcium/phosphorus ratio of the mineral removed from the enamel surfaces during demineralization, as well as the remineralization characteristics are similar [88]. Furthermore, caries progression in these two specimens is identical, and the inhibition and composition of biofilm formed are alike [89]. In addition, bovine enamel has approximately the same microhardness as human enamel [80], and no significant differences in bond strength between human and bovine enamel were found [90]. All in all, the reader must bear in mind the aforementioned differences and similarities when analyzing the results of the present review.

\section{Conclusions}

Resin infiltration significantly changes surface roughness, microhardness and shear bond strength in both sound enamel and WSLs. In sound enamel, infiltrative resins decrease $35 \%$ of surface roughness, $24 \%$ of microhardness and $25 \%$ of shear bond strength. In WSLs, enamel surface roughness reduced $54 \%$ after infiltrative resins application, but increased $68 \%$ and $89 \%$ its microhardness and shear bond strength, respectively. Furthermore, estimates point to an average penetration depth capacity of $65 \%$ in WSLs. These enamel characteristics can be affected by specimen, $\mathrm{pH}$, and etching time. Future studies with homogeneous methodologies are warranted to confirm these results.

Supplementary Materials: The following are available online at https://www.mdpi.com/article/10 $.3390 /$ jfb12030048/s1.

Author Contributions: Conceptualization, M.S., V.M., J.B. and C.M.; methodology, M.S., V.M. and C.M.; software, V.M. and J.B.; validation, M.S. and J.J.M.; formal analysis, V.M. and J.B.; investigation, M.S., V.M. and C.M.; resources, M.S.; data curation, M.S., V.M. and J.B.; writing-original draft preparation, M.S., V.M. and J.B.; writing—review and editing, J.B., V.M., C.M. and J.J.M.; visualization, J.B. and V.M.; supervision, V.M., C.M. and J.J.M. All authors have read and agreed to the published version of the manuscript.

Funding: This work is financed by national funds through the FCT-Foundation for Science and Technology, I.P., under the project UIDB/04585/2020.

Institutional Review Board Statement: Not applicable.

Informed Consent Statement: Not applicable.

Data Availability Statement: Data sharing is not applicable to this article.

Conflicts of Interest: The authors declare no conflict of interest. The funders had no role in the design of the study; in the collection, analyses, or interpretation of data; in the writing of the manuscript, or in the decision to publish the results.

\section{References}

1. Dorri, M.; Martinez-Zapata, M.J.; Walsh, T.; Marinho, V.C.; Sheiham deceased, A.; Zaror, C. Atraumatic Restorative Treatment versus Conventional Restorative Treatment for Managing Dental Caries. Cochrane Database Syst. Rev. 2017. [CrossRef]

2. Kassebaum, N.J.; Bernabé, E.; Dahiya, M.; Bhandari, B.; Murray, C.J.L.; Marcenes, W. Global Burden of Untreated Caries: A Systematic Review and Metaregression. J. Dent. Res. 2015, 94, 650-658. [CrossRef] [PubMed]

3. Bishara, S.E.; Ostby, A.W. White Spot Lesions: Formation, Prevention, and Treatment. Semin. Orthod. 2008, 14, 174-182. [CrossRef]

4. Heymann, G.C.; Grauer, D. A Contemporary Review of White Spot Lesions in Orthodontics: White Spot Lesions in Orthodontics. J. Esthet. Restor. Dent. 2013, 25, 85-95. [CrossRef] [PubMed]

5. Julien, K.C.; Buschang, P.H.; Campbell, P.M. Prevalence of White Spot Lesion Formation during Orthodontic Treatment. Angle Orthod. 2013, 83, 641-647. [CrossRef]

6. Sangamesh, B.; Kallury, A. Iatrogenic Effects of Orthodontic Treatment-Review on White Spot Lesions. Int. J. Sci. Eng. Res. 2011, 2, 2-16. 
7. Black, G.V. Operative Dentistry: The Pathology of the Hard Tissues of the Teeth, 1st ed.; Medico-Dnetal Publishing: Chicago, IL, USA, 1908.

8. Gorelick, L.; Geiger, A.M.; Gwinnett, A.J. Incidence of White Spot Formation after Bonding and Banding. Am. J. Orthod. 1982, 81, 93-98. [CrossRef]

9. Tufekci, E.; Dixon, J.S.; Gunsolley, J.C.; Lindauer, S.J. Prevalence of White Spot Lesions during Orthodontic Treatment with Fixed Appliances. Angle Orthod. 2011, 81, 206-210. [CrossRef]

10. Prevalence of White-Spot Lesions before and during Orthodontic Treatment with Fixed Appliances I European Journal of Orthodontics I Oxford Academic. Available online: https:/ /academic.oup.com/ejo/article/35/5/664/495801 (accessed on 12 June 2021).

11. Sundararaj, D.; Venkatachalapathy, S.; Tandon, A.; Pereira, A. Critical Evaluation of Incidence and Prevalence of White Spot Lesions during Fixed Orthodontic Appliance Treatment: A Meta-Analysis. J. Int. Soc. Prev. Community Dent. 2015, 5, 433-439. [CrossRef] [PubMed]

12. Urquhart, O.; Tampi, M.P.; Pilcher, L.; Slayton, R.L.; Araujo, M.W.B.; Fontana, M.; Guzmán-Armstrong, S.; Nascimento, M.M.; Nový, B.B.; Tinanoff, N.; et al. Nonrestorative Treatments for Caries: Systematic Review and Network Meta-Analysis. J. Dent. Res. 2019, 98, 14-26. [CrossRef]

13. Giudice, G.L.; Lipari, F.; Lizio, A.; Cervino, G.; Cicciù, M. Tooth Fragment Reattachment Technique on a Pluri Traumatized Tooth. J. Conserv. Dent. JCD 2012, 15, 80-83. [CrossRef]

14. Jobim Jardim, J.; Henz, S.; Barbachan, E.; Silva, B. Restorative Treatment Decisions in Posterior Teeth: A Systematic Review. Oral Health Prev. Dent. 2017, 15, 107-115. [CrossRef]

15. Abdullah, Z.; John, J. Minimally Invasive Treatment of White Spot Lesions-A Systematic Review. Oral Health Prev. Dent. 2016, 14, 197-205. [CrossRef]

16. Bhat, S.; Hegde, S.; Bhat, V.; Ramya, K.; Jodalli, P. Assessment of Maternal Risk Factors and Its Relationship with Early Childhood Caries among Preschool Children in Mangaluru City. J. Indian Soc. Pedod. Prev. Dent. 2017, 35, 193. [CrossRef]

17. Domöjean, S.; Ducamp, R.; Löger, S.; Holmgren, C. Resin Infiltration of Non-Cavitated Caries Lesions: A Systematic Review. Med. Princ. Pract. 2015, 24, 216-221. [CrossRef]

18. Meyer-Lueckel, H.; Paris, S.; Kielbassa, A.M. Surface Layer Erosion of Natural Caries Lesions with Phosphoric and Hydrochloric Acid Gels in Preparation for Resin Infiltration. Caries Res. 2007, 41, 223-230. [CrossRef] [PubMed]

19. Dorri, M.; Dunne, S.M.; Walsh, T.; Schwendicke, F. Micro-Invasive Interventions for Managing Proximal Dental Decay in Primary and Permanent Teeth. Cochrane Database Syst. Rev. 2015. [CrossRef] [PubMed]

20. Zakizade, M.; Davoudi, A.; Akhavan, A.; Shirban, F. Effect of Resin Infiltration Technique on Improving Surface Hardness of Enamel Lesions: A Systematic Review and Meta-Analysis. J. Evid. Based Dent. Pract. 2020, 20, 101405. [CrossRef] [PubMed]

21. Borges, A.B.; Caneppele, T.M.F.; Masterson, D.; Maia, L.C. Is Resin Infiltration an Effective Esthetic Treatment for Enamel Development Defects and White Spot Lesions? A Systematic Review. J. Dent. 2017, 56, 11-18. [CrossRef] [PubMed]

22. Aminoshariae, A.; Kulild, J. Master Apical File Size-Smaller or Larger: A Systematic Review of Microbial Reduction. Int. Endod. J. 2015, 48, 1007-1022. [CrossRef]

23. Dos Reis-Prado, A.H.; Abreu, L.G.; Tavares, W.L.F.; da Cunha Peixoto, I.F.; Viana, A.C.D.; de Oliveira, E.M.C.; Bastos, J.V.; Ribeiro-Sobrinho, A.P.; Benetti, F. Comparison between Immediate and Delayed Post Space Preparations: A Systematic Review and Meta-Analysis. Clin. Oral Investig. 2021, 25, 417-440. [CrossRef] [PubMed]

24. Ethem Yaylali, I.; Kececi, A.D.; Ureyen Kaya, B. Ultrasonically Activated Irrigation to Remove Calcium Hydroxide from Apical Third of Human Root Canal System: A Systematic Review of In Vitro Studies. J. Endod. 2015, 41, 1589-1599. [CrossRef]

25. Hozo, S.P.; Djulbegovic, B.; Hozo, I. Estimating the Mean and Variance from the Median, Range, and the Size of a Sample. BMC Med. Res. Methodol. 2005, 5, 13. [CrossRef] [PubMed]

26. Higgins, J.P.T.; Altman, D.G.; Gotzsche, P.C.; Juni, P.; Moher, D.; Oxman, A.D.; Savovic, J.; Schulz, K.F.; Weeks, L.; Sterne, J.A.C.; et al. The Cochrane Collaboration's Tool for Assessing Risk of Bias in Randomised Trials. BMJ 2011, 343 , d5928. [CrossRef]

27. AL Again, N.A.; Atta, M.S.; Alhady, A.A.A. The Effect of Caries Infiltration Technique (Icons) on Surface Roughness and Microhardness of Enamel White Spot Lesions. Al-Azhar J. Dent. Sci. 2018, 21, 79-86. [CrossRef]

28. Abdel-Hakim, S.; Metwalli, N.; El-Askary, F.; Wassel, M. Microhardness, sem and color change analysis of artificial enamel lesions in primary teeth treated with resin infiltration, cpp-acp or fluoride gel: An in vitro study. Egypt. Dent. J. 2016, 62, 3735-3744.

29. Abdellatif, A.; Shalan, H.; Elsebaai, A. Effects of Resin Application on White Spot Lesions (Laboratory Study). J. Clin. Pediatr. Dent. 2016, 46, 120-126.

30. Andrade Neto, D.M.; Carvalho, E.V.; Rodrigues, E.A.; Feitosa, V.P.; Sauro, S.; Mele, G.; Carbone, L.; Mazzetto, S.E.; Rodrigues, L.K.; Fechine, P.B.A. Novel Hydroxyapatite Nanorods Improve Anti-Caries Efficacy of Enamel Infiltrants. Dent. Mater. Off. Publ. Acad. Dent. Mater. 2016, 32, 784-793. [CrossRef] [PubMed]

31. Arslan, S.; Zorba, Y.O.; Atalay, M.A.; Özcan, S.; Demirbuga, S.; Pala, K.; Percin, D.; Ozer, F. Effect of Resin Infiltration on Enamel Surface Properties and Streptococcus Mutans Adhesion to Artificial Enamel Lesions. Dent. Mater. J. 2015, 34, 25-30. [CrossRef]

32. Attin, R.; Stawarczyk, B.; Keçik, D.; Knösel, M.; Wiechmann, D.; Attin, T. Shear Bond Strength of Brackets to Demineralize Enamel after Different Pretreatment Methods. Angle Orthod. 2012, 82, 56-61. [CrossRef] 
33. Ayad, A.; Mustafa, D.; Nour, K. Effect of Remineralizing Agents and Resin Infiltration on Resistance to Demineralization of Artificial Enamel Lesions. Egypt. Dent. J. 2020, 66, 2763-2771. [CrossRef]

34. Aziznezhad, M.; Alaghemand, H.; Shahande, Z.; Pasdar, N.; Bijani, A.; Eslami, A.; Dastan, Z. Comparison of the Effect of Resin Infiltrant, Fluoride Varnish, and Nano-Hydroxy Apatite Paste on Surface Hardness and Streptococcus Mutans Adhesion to Artificial Enamel Lesions. Electron. Physician 2017, 9, 3934-3942. [CrossRef]

35. Behrouzi, P.; Heshmat, H.; Hoorizad Ganjkar, M.; Tabatabaei, S.F.; Kharazifard, M.J. Effect of Two Methods of Remineralization and Resin Infiltration on Surface Hardness of Artificially Induced Enamel Lesions. J. Dent. Shiraz Iran 2020, 21, 12-17. [CrossRef]

36. El Meligy, O.A.E.S.; Alamoudi, N.M.; Eldin Ibrahim, S.T.; Felemban, O.M.; Al-Tuwirqi, A.A. Effect of Resin Infiltration Application on Early Proximal Caries Lesions in Vitro. J. Dent. Sci. 2021, 16, 296-303. [CrossRef]

37. Enan, E.T.; Tawfik, M.A.; Ali, A.I.; Anan, A.T. Surface properties of resin-infiltrated incipient enamel lesions after aging under thermal stresses. Egypt. Dent. J. 2018, 64, 2833-2840. [CrossRef]

38. Gurdogan, E.B.; Ozdemir-Ozenen, D.; Sandalli, N. Evaluation of Surface Roughness Characteristics Using Atomic Force Microscopy and Inspection of Microhardness Following Resin Infiltration with Icon ${ }^{\circledR}$. J. Esthet. Restor. Dent. Off. Publ. Am. Acad. Esthet. Dent. Al 2017, 29, 201-208. [CrossRef]

39. Horuztepe, S.A.; Baseren, M. Effect of Resin Infiltration on the Color and Microhardness of Bleached White-Spot Lesions in Bovine Enamel (an in vitro Study). J. Esthet. Restor. Dent. Off. Publ. Am. Acad. Esthet. Dent. Al 2017, 29, 378-385. [CrossRef]

40. Mandava, J.; Reddy, Y.S.; Kantheti, S.; Chalasani, U.; Ravi, R.C.; Borugadda, R.; Konagala, R.K. Microhardness and Penetration of Artificial White Spot Lesions Treated with Resin or Colloidal Silica Infiltration. J. Clin. Diagn. Res. JCDR 2017, 11, ZC142-ZC146. [CrossRef]

41. Montasser, M.A.; El-Wassefy, N.A.; Taha, M. In Vitro Study of the Potential Protection of Sound Enamel against Demineralization. Prog. Orthod. 2015, 16, 12. [CrossRef]

42. Pancu, G.; Andrian, S.; Iovan, G.; Ghiorghe, A.; Topoliceanu, C.; Moldovanu, A.; Georgescu, A.; Pancu, I.; Stoleriu, S. Study regarding the assessment of enamel microhardness in incipient carious lesions treated by icon method. Rom. J. Oral Rehabil. 2011, $3,94-100$.

43. Paris, S. Micro-Hardness and Mineral Loss of Enamel Lesions after Infiltration with Various Resins: Influence of Infiltrant Composition and Application Frequency in Vitro-ScienceDirect. Available online: https://www.sciencedirect.com/science/ article/abs / pii/S0300571213000869 (accessed on 10 March 2021).

44. Prajapati, D.; Nayak, R.; Pai, D.; Upadhya, N.; Bhaskar, V.K.; Kamath, P. Effect of Resin Infiltration on Artificial Caries: An in Vitro Evaluation of Resin Penetration and Microhardness. Int. J. Clin. Pediatr. Dent. 2017, 10, 250-256. [CrossRef] [PubMed]

45. Qasim, A. Assessement of White Spot Treated with (ICON) and Flouride Gel (An In Vitro Study). Int. J. Enhanc. Res. Sci. Technol. Eng. 2014, 3, 1-6.

46. El-zankalouny Shimaa, M.; Abd El Fattah Wegdan, M.; El-Shabrawy Sonia, M. Penetration depth and enamel microhardness of resin infiltrant and traditional techniques for treatment of artificial enamel lesions. Alex. Dent. J. 2016, 41, 20-25. [CrossRef]

47. Taher, N.M.; Alkhamis, H.A.; Dowaidi, S.M. The Influence of Resin Infiltration System on Enamel Microhardness and Surface Roughness: An in Vitro Study. Saudi Dent. J. 2012, 24, 79-84. [CrossRef] [PubMed]

48. Torres, C.R.G.; Rosa, P.C.F.; Ferreira, N.S.; Borges, A.B. Effect of Caries Infiltration Technique and Fluoride Therapy on Microhardness of Enamel Carious Lesions. Oper. Dent. 2012, 37, 363-369. [CrossRef] [PubMed]

49. Yazkan, B.; Ermis, R.B. Effect of Resin Infiltration and Microabrasion on the Microhardness, Surface Roughness and Morphology of Incipient Carious Lesions. Acta Odontol. Scand. 2018, 76, 473-481. [CrossRef] [PubMed]

50. Arora, T.C.; Arora, D.; Tripathi, A.M.; Yadav, G.; Saha, S.; Dhinsa, K. An In-Vitro Evaluation of Resin Infiltration System and Conventional Pit and Fissure Sealant on Enamel Properties in White Spot Lesions. J. Indian Soc. Pedod. Prev. Dent. 2019, 37, 133-139. [CrossRef]

51. Aswani, R.; Chandrappa, V.; Uloopi, K.; Chandrasekhar, R.; RojaRamya, K.S. Resin Infiltration of Artificial Enamel Lesions: Evaluation of Penetration Depth, Surface Roughness and Color Stability. Int. J. Clin. Pediatr. Dent. 2019, 12, 520-523. [CrossRef]

52. Baka, Z.M.; Akin, M.; Ileri, Z.; Basciftci, F.A. Effects of Remineralization Procedures on Shear Bond Strengths of Brackets Bonded to Demineralized Enamel Surfaces with Self-Etch Systems. Angle Orthod. 2016, 86, 661-667. [CrossRef]

53. Enan, E.T.; Aref, N.S.; Hammad, S.M. Resistance of Resin-Infiltrated Enamel to Surface Changes in Response to Acidic Challenge. J. Esthet. Restor. Dent. Off. Publ. Am. Acad. Esthet. Dent. Al 2019, 31, 353-358. [CrossRef]

54. Khalid, I.; Anggani, H.S.; Ismah, N. Differences in Enamel Surface Roughness Changes after Debonding Using Resin Infiltration System and Nano-Filled Resin Coating. J. Int. Dent. Med. Res. 2019, 12, 95-100.

55. Askar, H.; Schwendicke, F.; Lausch, J.; Meyer-Lueckel, H.; Paris, S. Modified Resin Infiltration of Non-, Micro- and Cavitated Proximal Caries Lesions in Vitro. J. Dent. 2018, 74, 56-60. [CrossRef]

56. Gelani, R.; Zandona, A.F.; Lippert, F.; Kamocka, M.M.; Eckert, G. In Vitro Progression of Artificial White Spot Lesions Sealed with an Infiltrant Resin. Oper. Dent. 2014, 39, 481-488. [CrossRef]

57. Lausch, J.; Paris, S.; Selje, T.; Dörfer, C.E.; Meyer-Lueckel, H. Resin Infiltration of Fissure Caries with Various Techniques of Pretreatment in Vitro. Caries Res. 2015, 49, 50-55. [CrossRef] [PubMed] 
58. López López, E.A.; Dominguez, J.A.; Gomes, G.M.; Mora, C.A.P.; Bittencourt, B.F.; Gomes, J.C.; Gomes, O.M.M. Effect of Conditioning Protocols and Ultrasonic Application of an Infiltrant Resin in White Spot Lesions. Braz. Dent. J. 2019, $30,58-65$. [CrossRef]

59. Meyer-Lueckel, H.; Chatzidakis, A.; Naumann, M.; Dörfer, C.E.; Paris, S. Influence of Application Time on Penetration of an Infiltrant into Natural Enamel Caries. J. Dent. 2011, 39, 465-469. [CrossRef] [PubMed]

60. Min, J.H.; Inaba, D.; Kwon, H.K.; Chung, J.H.; Kim, B.I. Evaluation of Penetration Effect of Resin Infiltrant Using Optical Coherence Tomography. J. Dent. 2015, 43, 720-725. [CrossRef]

61. Paris, S.; Soviero, V.M.; Schuch, M.; Meyer-Lueckel, H. Pretreatment of Natural Caries Lesions Affects Penetration Depth of Infiltrants in Vitro. Clin. Oral Investig. 2013, 17, 2085-2089. [CrossRef]

62. Paris, S.; Bitter, K.; Naumann, M.; Dörfer, C.E.; Meyer-Lueckel, H. Resin Infiltration of Proximal Caries Lesions Differing in ICDAS Codes. Eur. J. Oral Sci. 2011, 119, 182-186. [CrossRef] [PubMed]

63. Paris, S.; Lausch, J.; Selje, T.; Dörfer, C.E.; Meyer-Lueckel, H. Comparison of Sealant and Infiltrant Penetration into Pit and Fissure Caries Lesions in Vitro. J. Dent. 2014, 42, 432-438. [CrossRef]

64. Paris, S.; Soviero, V.M.; Seddig, S.; Meyer-Lueckel, H. Penetration Depths of an Infiltrant into Proximal Caries Lesions in Primary Molars after Different Application Times in Vitro. Int. J. Paediatr. Dent. 2011, 22, 349-355. [CrossRef]

65. Sava-rosianu, R.; Podariu, A.; Negrutiu, M.; Szuhanek, C.; Sinescu, C. Low Viscosity Resin Penetration Degree in Incipient Caries Lesions. Rev. Chim. 2017, 68, 2588-2592. [CrossRef]

66. Theodory, T.G.; Kolker, J.L.; Vargas, M.A.; Maia, R.R.; Dawson, D.V. Masking and Penetration Ability of Various Sealants and ICON in Artificial Initial Caries Lesions In Vitro. J. Adhes. Dent. 2019, 21, 265-272. [CrossRef] [PubMed]

67. Wang, L.; de Almendra Freitas, M.C.C.; Prakki, A.; Mosquim, V.; González, A.H.M.; Rios, D.; Honório, H.M. Experimental Self-Etching Resin Infiltrants on the Treatment of Simulated Carious White Spot Lesions. J. Mech. Behav. Biomed. Mater. 2021, 113, 104146. [CrossRef] [PubMed]

68. Borges, A.B.; Abu Hasna, A.; Matuda, A.G.N.; Lopes, S.R.; Mafetano, A.P.V.P.; Arantes, A.; Duarte, A.F.; Barcellos, D.C.; Torres, C.R.G.; Pucci, C.R. Adhesive Systems Effect over Bond Strength of Resin-Infiltrated and de/Remineralized Enamel. F1000Research 2019, 8, 1743. [CrossRef]

69. Dilber, E.; Akın, M.; Yavuz, T.; Erdem, A. Effects of Different Demineralization-Inhibiting Methods on the Shear Bond Strength of Glass-Ceramics. J. Prosthodont. Off. J. Am. Coll. Prosthodont. 2015, 24, 407-413. [CrossRef] [PubMed]

70. Ekizer, A.; Zorba, Y.O.; Uysal, T.; Ayrikcila, S. Effects of Demineralizaton-Inhibition Procedures on the Bond Strength of Brackets Bonded to Demineralized Enamel Surface. Korean J. Orthod. 2012, 42, 17-22. [CrossRef] [PubMed]

71. Gulec, A. Assessment of the Resin Infiltration and CPP-ACP Applications before Orthodontic Brackets Bonding-PubMed. Available online: https:/ / pubmed.ncbi.nlm.nih.gov/31434834/ (accessed on 11 March 2021).

72. Velİ, I.; Akin, M.; Baka, Z.M.; Uysal, T. Effects of Different Pre-Treatment Methods on the Shear Bond Strength of Orthodontic Brackets to Demineralized Enamel. Acta Odontol. Scand. 2016, 74, 7-13. [CrossRef]

73. Vianna, J.S.; Marquezan, M.; Lau, T.C.L.; Sant'Anna, E.F. Bonding Brackets on White Spot Lesions Pretreated by Means of Two Methods. Dent. Press J. Orthod. 2016, 21, 39-44. [CrossRef]

74. Paris, S.; Schwendicke, F.; Keltsch, J.; Dörfer, C.; Meyer-Lueckel, H. Masking of White Spot Lesions by Resin Infiltration in Vitro. J. Dent. 2013, 41, e28-e34. [CrossRef] [PubMed]

75. Paris, S.; Meyer-Lueckel, H. Resin Infiltration after Enamel Etching. In Tooth Whitening; Perdigão, J., Ed.; Springer International Publishing: Cham, Switzerland, 2016; pp. 211-222. ISBN 978-3-319-38847-2.

76. Mohamed, A.M.; Wong, K.H.; Lee, W.J.; Marizan Nor, M.; Mohd Hussaini, H.; Rosli, T.I. In Vitro Study of White Spot Lesion: Maxilla and Mandibular Teeth. Saudi Dent. J. 2018, 30, 142-150. [CrossRef]

77. Attia, R.M. Effect of PH Challenge on the Micro-Hardness of Artificially Induced de-Mineralized Enamel Treated with Resin Infiltrant and Fluoride Varnish. Egypt. Dent. J. 2018, 64, 2841-2850. [CrossRef]

78. Wong, A.; Subar, P.E.; Young, D.A. Dental Caries. Adv. Pediatr. 2017, 64, 307-330. [CrossRef] [PubMed]

79. Abou Neel, E.A.; Aljabo, A.; Strange, A.; Ibrahim, S.; Coathup, M.; Young, A.M.; Bozec, L.; Mudera, V. DemineralizationRemineralization Dynamics in Teeth and Bone. Int. J. Nanomed. 2016, 11, 4743-4763. [CrossRef] [PubMed]

80. Ten Cate, J.M.; Featherstone, J.D.B. Mechanistic Aspects of the Interactions Between Fluoride and Dental Enamel. Crit. Rev. Oral Biol. Med. 1991, 2, 283-296. [CrossRef]

81. Neres, É.; Moda, M.; Chiba, E.; Briso, A.; Pessan, J.; Fagundes, T. Microhardness and Roughness of Infiltrated White Spot Lesions Submitted to Different Challenges. Oper. Dent. 2017, 42, 428-435. [CrossRef] [PubMed]

82. Rasmussen, S.T. Analysis of Dental Shear Bond Strength Tests, Shear or Tensile? Int. J. Adhes. Adhes. 1996, 16, 147-154. [CrossRef]

83. Nakabayashi, N.; Nakamura, M.; Yasuda, N. Hybrid Layer as a Dentin-Bonding Mechanism. J. Esthet. Dent. 1991, 3, 133-138. [CrossRef]

84. Perdigão, J. Resin Infiltration of Enamel White Spot Lesions: An Ultramorphological Analysis. J. Esthet. Restor. Dent. Off. Publ. Am. Acad. Esthet. Dent. Al 2020, 32,317-324. [CrossRef]

85. Yassen, G.H.; Platt, J.A.; Hara, A.T. Bovine Teeth as Substitute for Human Teeth in Dental Research: A Review of Literature. J. Oral Sci. 2011, 53, 273-282. [CrossRef]

86. Arends, J.; Jongebloed, W.L. Crystallites Dimensions of Enamel. J. Biol. Buccale 1978, 6, 161-171. 
87. Mellberg, J.R. Hard-Tissue Substrates for Evaluation of Cariogenic and Anti-Cariogenic Activity in Situ. J. Dent. Res. 1992, 71, 913-919. [CrossRef] [PubMed]

88. Feagin, F.; Koulourides, T.; Pigman, W. The Characterization of Enamel Surface Demineralization, Remineralization, and Associated Hardness Changes in Human and Bovine Material. Arch. Oral Biol. 1969, 14, 1407-1417. [CrossRef]

89. Hara, A.T.; Queiroz, C.S.; Paes Leme, A.F.; Serra, M.C.; Cury, J.A. Caries Progression and Inhibition in Human and Bovine Root Dentine in Situ. Caries Res. 2003, 37, 339-344. [CrossRef]

90. Rüttermann, S.; Braun, A.; Janda, R. Shear Bond Strength and Fracture Analysis of Human vs. Bovine Teeth. PLoS ONE 2013, 8 , e59181. [CrossRef] [PubMed] 